\title{
Multi-Supervisory Control and Data Display
}

\author{
Rajeev Kumar \\ Senior Lecturer \\ GCET \\ Greater Noida, India
}

\author{
M.L.Dewal \\ Faculty \\ IIT Roorkee \\ Roorkee, India
}

\begin{abstract}
Supervisory control and data acquisition (SCADA) is a system that allows an operator to monitor and control processes that are distributed among various remote sites. SCADA is a system in which message or commands that are individual are sends to the external world. This document provides an overview of the multi supervisory control and Data Display in Supervisory Control and Data Acquisition (SCADA) and other Process Control Systems .The goal of this paper to make our system flexible, reliable and minimize the cable cost. This document also provides PLC interlocking and real time the data sharing.
\end{abstract}

\section{Keywords}

SCADA, PLC, HMI

\section{INTRODUCTION}

Process Control Systems (PCS) refer to the overall set of systems that remotely monitor and measure remote sensors from a centralized location. These sensors also typically possess some type of automated response capability when certain criteria are met [4].

A subset of PCS systems that manage systems over very large geographic areas are typically referred to as Supervisory Control and Data Acquisition systems or SCADA systems. SCADA systems make up the critical infrastructure associated with electric utilities, water and sewage treatment plants, and largescale transportation systems like interstate rail.

Supervisory control and data acquisition (SCADA) is a system that allows an operator to monitor and control processes that are distributed among various remote sites. There are many processes that use SCADA systems: hydroelectric, water distribution and treatment utilities, natural gas, etc. SCADA systems allow remote sites to communicate with a control facility and provide the necessary data to control processes.

For many of its uses, SCADA provides an economic advantage. As the distance and inaccessibility to remote sites increases, SCADA becomes a better alternative than an operator or repairman's visiting the site for adjustments and inspections. Distance and accessibility are two major factors for implementing SCADA systems. SCADA can be traced to the development of telemetry from the first half of the century.
Telemetry is the transmission and collection of data obtained by sensing real-time conditions. The technology of rockets and aircraft afforded man with the opportunity to investigate weather and planetary data. This required a simple way to get data from space that observers could not normally achieve. Manned stations on the surface of the Earth such as lighthouses, post offices, weather stations, etc., were able to collect and monitor data on weather.

\section{A SCADA system performs four basic functions: \\ 1. Data acquisition \\ 2. Networked data communication \\ 3. Data presentation \\ 4. Control}

These functions are performed by four kinds of SCADA components:

1.Sensors (either digital or analog) and control relays that directly interface with the managed system.

2. Remote Terminal Units (RTUs) [5]: Remote terminal units gather information from their remote site from various input devices, like valves, pumps, alarms, meters, etc. Essentially, data is either analog (real numbers), digital (on/off), or pulse data (e.g., counting the revolutions of a meter). Many remote terminal units hold the information gathered in their memory and wait for a request from the MTU to transmit the data. Other more sophisticated remote terminal units have microcomputers and programmable logic controllers (PLC) that can perform direct control over a remote site without the direction of the MTU. Figure 2 shows an example of outputs of the RTU to the MTU and to the field devices.

3. Master Terminal Units (MTU) [5]: The master terminal unit initiates all communication, gathers data, stores information, sends information to other systems, and interfaces with operators. The major difference between the MTU and RTU is that the MTU initiates virtually all communications between the two. The MTU also communicates with other peripheral devices in the facility like monitors, printers, and other information 
systems. The primary interface to the operator is the monitor or CRT that portrays a representation of valves, pumps, etc. As incoming data changes, the screen is updated.

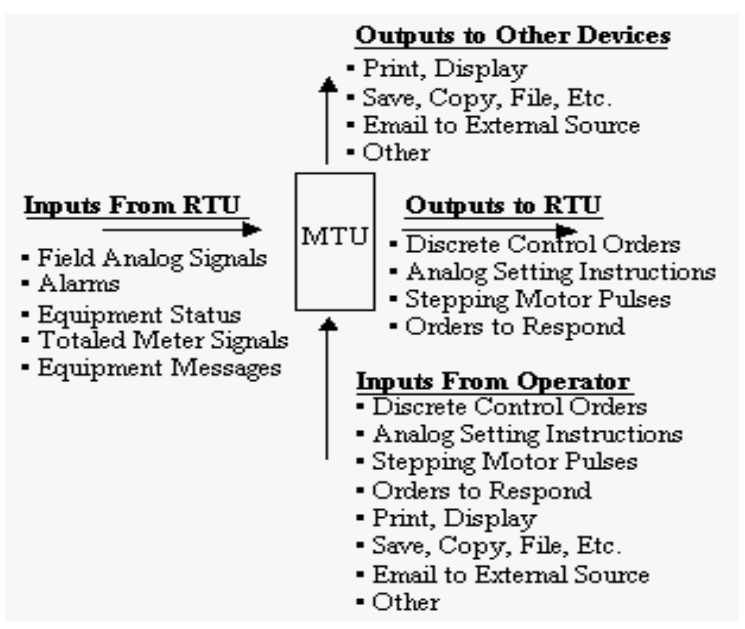

Figure1.Inputs and Outputs of an MTU [5]

4. The Communications Equipments [5]: Communication equipments are the devices which are used to connect the SCADA master unit to the RTUs in the field.

Communication equipment is required for bi-directional communications between an RTU and the MTU. This can be done through public transmission media or atmospheric means. SCADA systems are capable of communicating using a wide variety of media such as fiber optics, dial-up, or dedicated voice grade telephone lines, or radio. Recently, some utilities have employed Integrated Services Digital Network (ISDN). Since the amount of information transmitted are relatively small (less than $50 \mathrm{~K})$, voice grade phone lines, and radio work well.

SCADA system owners bear the ultimate responsibility for protecting what they manage. They have participated in vulnerability assessments, have made improvements and continue to do so. Through vulnerability assessments and responding to research questions, they also provide the information that gives direction for other stakeholders.

SCADA can use to manage any kind of equipment. Typically, SCADA systems are used to automate complex industrial processes where human control is impractical. Systems where there are more control factors, and more fast-moving control factors, than human beings can comfortably manage. Around the world, SCADA systems control:

- Water and sewage: State and municipal water utilities use SCADA to monitor and regulate water flow, reservoir levels, pipe pressure and other factors.
- Buildings, facilities and environments: Facility managers use SCADA to control HVAC, refrigeration units, lighting and entry systems.

- Manufacturing: SCADA systems manage parts inventories for just-in-time manufacturing, regulate industrial automation and robots, and monitor process and quality control.

Here are few of the things you can do with the information and control capabilities you get from a SCADA system:

- Access quantitative measurements of important processes, both immediately and over time

- Detect and correct problems as soon as they begin

- Measure trends over time

- Discover and eliminate bottlenecks and inefficiencies

- Control larger and more complex processes with a smaller, less specialized staff

Supervisory control and data acquisition (SCADA) allows a utility operator to monitor and control processes that are distributed among various remote sites. The goal of this paper is to develop a management paper work that uses existing probabilistic control of production methodology to quantify the Number of panel of the plant to production utility SCADA systems. SCADA system owners bear the ultimate responsibility for protecting what they manage. They have participated in vulnerability assessments, have made improvements and continue to do so. Through vulnerability assessments and responding to research questions, they also provide the information that gives direction for other stakeholders.

SCADA have a wide variety of functions which are crucial to the day-to-day running of electrical power utility. These functions include identifying faults, isolating them and restoring service, circuit breaker and re-closer control, feeder switching, voltage regulator, monitoring, transformer temperature, and metering.

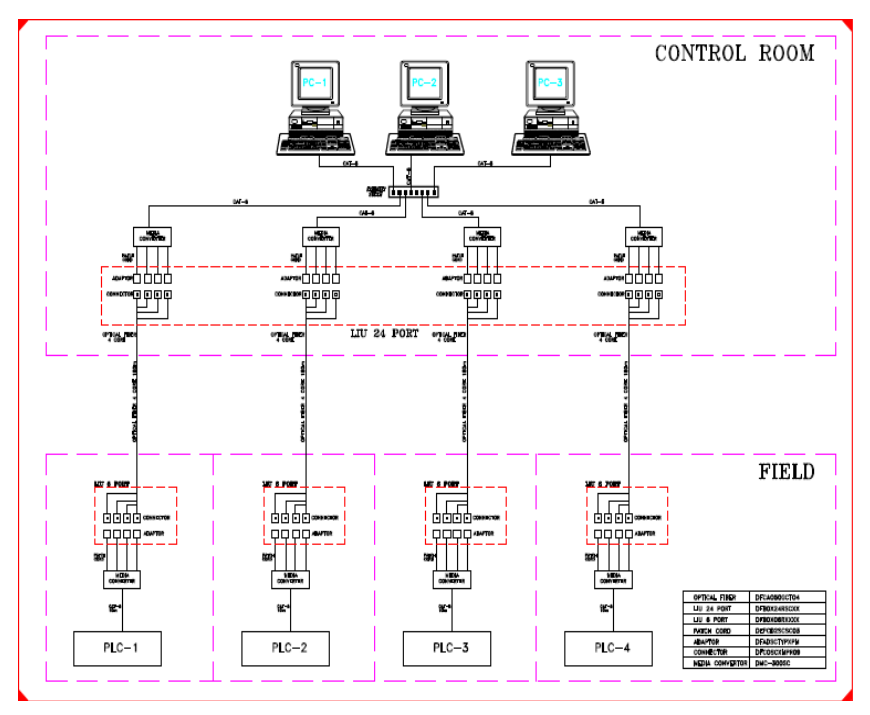




\section{EXPERIMENTAL SET-UP}

\subsection{Hardware}

Figure 2.Layout of Multi Supervisory Control and Data display

We have implement a SCADA in a water treatment plant. We control the water treatment process from multi-places and display all the plant sites at different places i.e. collect different sites at different places. the layout of this system is shown in figure 2. To minimize complex ability of the network and the cable, we placed separate PLC at each site and these all PLCs are connected to the multi-MTU by the optical fiber (DFCAO50GCTO4). There are four PLC Called PLC-1,PLC2,PLC-3,PLC-4 are placed at different sites site-1, site-2, site-3, site- 4 respectively of the plant and reports the each site status to the computer or control room by the optical fiber 4 core $180 \mathrm{Cm}$ cable. We use optical fiber because of the following advantages of the optical fiber:

1. Fiber optic cables have a much greater bandwidth than metal cables. This means that they can carry more data.

2. Fiber optic cables are less susceptible than metal cables to interference.

3. Fiber optic cables are much thinner and lighter than metal wires.

4. Data can be transmitted digitally (the natural form for computer data) rather than analogically.

The illustration indicates the source-user pair, transmitter and receiver. It also clearly shows the fiber optic cable constituting the transmission medium as well as the connectors that provide the interface of the transmitter to the transmission medium and transmission medium to the receiver.

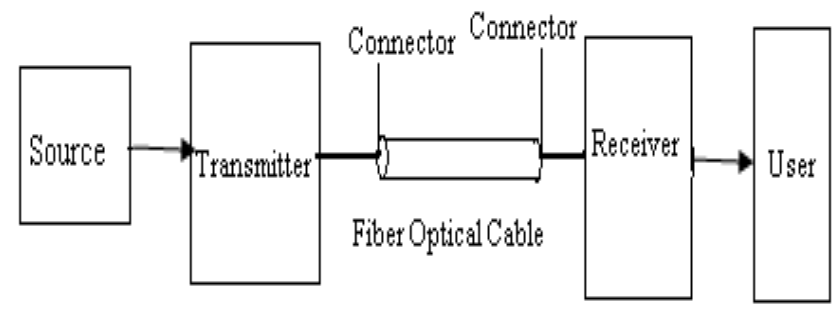

Figure3. Model of simple fiber optic data link

Media converter is a device which converts the one type of media to another i.e. twisted pair to optical fiber. Here media converter converts the electrical signal to the light form. A patch cord is a piece of Copper wire or fiber optic cable that connects circuits on a patch panel. It is also known as a patch cable.

Fiber optic is a technology that uses glass (or plastic ) threads (fibers) to transmit data. A fiber optic cable consists of a bundle of glass threads, each of which is capable of transmitting messages modulated onto light waves. fiber optics is a particularly popular technology for LAN. In addition, telephone comprises are steadily replacing traditional telephone lines with fiber optic cables. In the future, almost all communications will employ fiber optic.

\subsection{Software}

Software products typically used within a SCADA system are as follows:

- Central host computer operating system: Software used to control the central host computer hardware. The software can be based on UNIX or other popular operating systems.

- Operator terminal operating system: Software used to control the central host computer hardware. The software is usually the same as the central host computer operating system. This software, along with that for the central host computer, usually contributes to the networking of the central host and the operator terminals.

- Central host computer application: Software that handles the transmittal and reception of data to and from the RTUs and the central host. The software also provides the graphical user interface which offers site mimic screens, alarm pages, trend pages, and control functions.

- Operator terminal application: Application that enables users to access information available on the central host computer application. It is usually a subset of the software used on the central host computers.

- Communications protocol drivers: Software that is usually based within the central host and the RTUs, and is required to control the translation and interpretation of the data between ends of the communications links in the system. The protocol drivers prepare the data for use either at the field devices or the central host end of the system.

- Communications network management software: Software required to control the communications network and to allow the communications networks them selves to be monitored for performance and failures.

- $\quad$ RTU automation software: Software that allows engineering staff to configure and maintain the application housed within the RTUs (or PLCs). Most often this includes the local automation application and any data processing tasks that are performed within the RTU.

An important aspect of every SCADA system is the computer software used within the system. The most obvious software component is the operator interface or Man Machine Interface/Human Machine Interface (MMI/HMI) package; however, software of some form pervades all levels of a SCADA system. Depending on the size and nature of the SCADA application, software can be a significant cost item when developing, maintaining, and expanding a SCADA system. When software is well defined, designed, written, checked, and tested, a successful SCADA system will likely be produced. Poor 
performances in any of these project phases will very easily because a SCADA project to fail.

We develop a SCADA window to collect all the four sites of the plant. i.e complete plant controlling and monitoring system as shown in fig.4. Plant sites are connected to the SCADA server via PLC. Programmable logic controller collects the data from the hardware and all this information's send to the SCADA server.

\section{OPERATION}

We installed a SCADA system for the water plant shown in fig.4. First the water enter to the INLET chamber from the raw effluent from collection system and sludge drying bed and other plant recirculation. There are four stage of water storage INLET chamber, raw effluent sump/pumping station, distribution chamber and then grit chamber.

There is a mechanical coarse screen between the INLET chamber and the raw effluent sump/pumping station. which is consists of two level switches(LS-206,LS-207), two pumps (CSM-C,CSMD) and pump Cm-B, level switch LS-206 and LS-20 show the status of the water level in the line 1 and line 2 respectively. Pump CSM-C, CSM-D are used to displacement in the mechanical coarse screen. These mechanical screens are also connected to another pump CM-B. CM-B is used to eliminate the coarse from both the screen to outside disposal.

Raw effluent sump/pumping station consist of three submersible pump A, B, C and level switch LS200 and level transmitter Lt 201. The level transmitter shows the water level in the raw effluent sump/pumping station and effect the operation of the submersible pumps. There is also a flow transmitter FT201 which shows the flow reading in $\mathrm{M}^{3} / \mathrm{h}$ and activates the relative alarm.

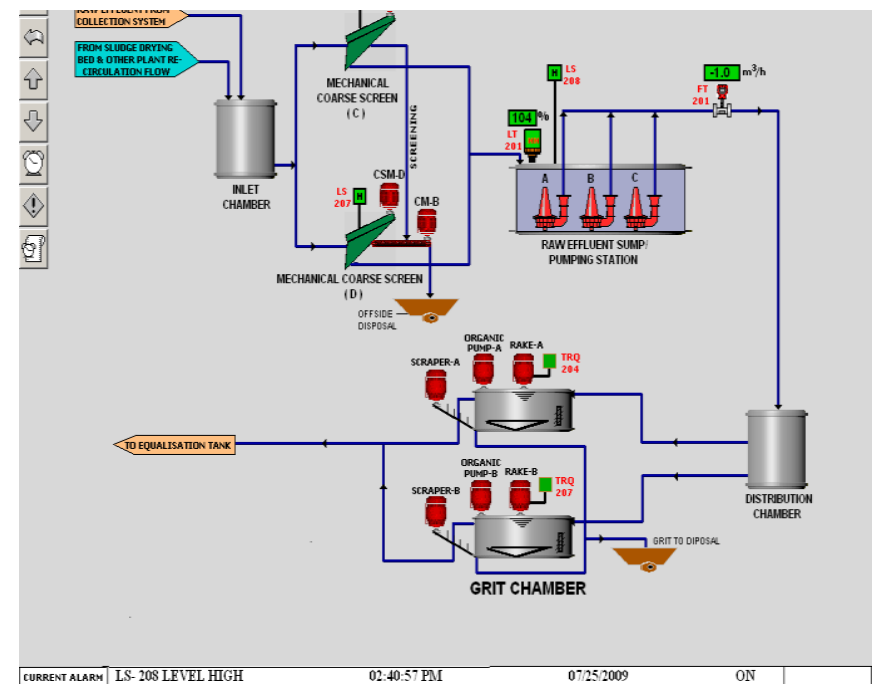

Figure.4. SCADA screen showing status of a raw effluent system
Now waste water transfer from dustbin chamber to grit chamber. In the grit chamber Rack-A and Rack-B rotate (Blower) the water. Scraper-A and Scraper-B eliminate or push the grit to the disposal from the water. Organic pump A and organic Pump B, pump the coarse, Stone and dust free water to the equalisation tank.

\section{RESULTS}

The alarm status with time and date is shown in fig.5. the status of these alarm also show to the user in real run time operation as shown in the fig. 4 .

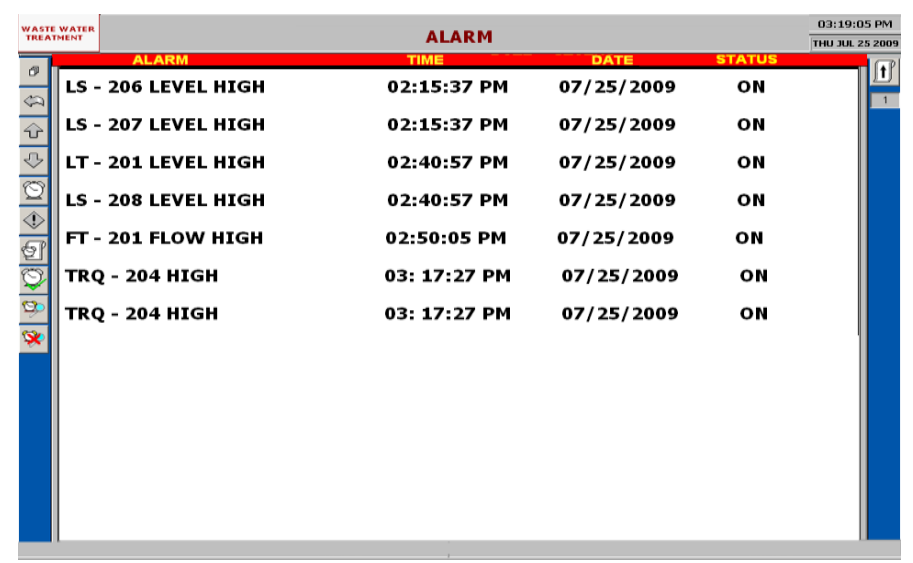

Figure5. Status of alarms

\section{CONCLUSIONS AND DISCUSSIONS}

This paper presents a SCADA system for raw effluent system. There is fulfilment of two tasks with the help of SCADA.

First it shows the status of the plant in real time and if there is a change in any one of the parameter it shows the value of the parameter. If the parameter is out of range then SCADA activate the respective alarm as shown an alarm "LS-206 LEVEL HIGH" in the figure 4 .

This paper also present Multi- Supervisory Control and data display as shown in fig.2. Same SCADA software is installed in the three computers or HMI and all these computers are connected to the all plant sites. This provides the multi supervisory control and data display, gives the user security because if the user feed any one of the parameter then the same value will be shown at all the remaining computers in real time.

This system also provides a flexible and reliable system and minimizes the initial cost of the system.

\section{REFERENCES}

[1]Bruce Li, P.Eng. "SCADA application in water and waste water industry" Declan IWS, February, 2007.

[2]Supervisory control and data Acquisition (SCADA) system, National Communication system Technical information bulletin 04-1, pages 4-9, October 2004. 
[3] "A Strategic Approach to Protecting SCADA and Process Control Systems”, IBIM Internet Security Systems white paper, 2007 Pages: 2.

[4] Ezell, Barry, "Supervisory Control And Data Acquisition Systems for Water Supply and Its Vulnerability to Cyber Risks" available on the internet at: http://watt.seas.virginia.edu/ bce4k/home.html. (August 1997).

[5] "Trends in SCADA for Automated water Systems"Synchrony 6410 Commonwealth Drive Roanoke, Virginia 24018, page2-7.

[6] Ezell, Barry, "Scenarios One and Two: Source to No1 PS to No 1 Tank to No 2 PS to No 2 tank (High level) for a MasterSlave SCADA System", SCADA Consultants,SCADA Mail List, scada@gospel.iinet.au (August

1997).

[7] Walski, Thomas M., et. al., Advanced Water Distribution
Modeling and Management, Haestad Press, January 2003.

[8] “Allen Bradley", 747 Alpha Drive, Highland Heights, OH 44143, USA.

[9] "Electronic Industries Association (EIA)”, 2001 I Street NW, Washington, DC 20006, USA.

[10]__Institute of Electrical and Electronic Engineers (IEEE)”, 345 East 47th St., New York, NY 10017, USA.

[11]McClanahan, R.H., "The Benefits of Networked SCADA Systems Utilizing IP- Enabled Networks" Rural Electric Power Conference, 2002. 2002 IEEE, 5-7 May 2002 Pages: C5 - C5_7

[12] __ IEEE Recommended Practice for "Data Communications between Remote Terminal Units and Intelligent Electronic Devices in a Substation", IEEE Std 1379- 2000 (Revision of IEEE Std 1379-1997), 21 September 2000 